\title{
Efficacy and Safety of Radiotherapy Plus Anti-PD1 Versus Transcatheter Arterial Chemoembolization Plus Sorafenib For Advanced Hepatocellular Carcinoma: a Real-World Study
}

\section{Jian-Xu Li ( $\square$ lijianxu1236@163.com )}

Guangxi Cancer Hospital and Guangxi Medical University Affiliated Cancer Hospital https://orcid.org/0000-0002-5298-3673

\section{Wen-Xiang Deng}

Guangxi Cancer Hospital and Guangxi Medical University Affiliated Cancer Hospital Shi-Ting Huang

Guangxi Cancer Hospital and Guangxi Medical University Affiliated Cancer Hospital Xiao-Feng Lin

Guangxi Cancer Hospital and Guangxi Medical University Affiliated Cancer Hospital

\section{Mei-Ying Long}

Guangxi Medical University

Jie Zhang

Guangxi Cancer Hospital and Guangxi Medical University Affiliated Cancer Hospital

\section{Ting-Shi Su}

Guangxi Cancer Hospital and Guangxi Medical University Affiliated Cancer Hospital

\section{Li-Qing Li}

Guangxi Cancer Hospital and Guangxi Medical University Affiliated Cancer Hospital

\section{Ya-Dan Pang}

Guangxi Cancer Hospital and Guangxi Medical University Affiliated Cancer Hospital Chun-Feng Liang

Guangxi Cancer Hospital and Guangxi Medical University Affiliated Cancer Hospital Hong-Mei Zhou

Guangxi Cancer Hospital and Guangxi Medical University Affiliated Cancer Hospital Hai-Yan Lu

Guangxi Cancer Hospital and Guangxi Medical University Affiliated Cancer Hospital Shi-Xiong Liang

Guangxi Cancer Hospital and Guangxi Medical University Affiliated Cancer Hospital Bang-De Xiang

Guangxi Cancer Hospital and Guangxi Medical University Affiliated Cancer Hospital 


\section{Research Article}

Keywords: hepatocellular carcinoma, anti-PD1, radiotherapy, overall survival, transcatheter arterial chemoembolization, sorafenib

Posted Date: December 29th, 2021

DOI: https://doi.org/10.21203/rs.3.rs-1190210/v1

License: (c) (i) This work is licensed under a Creative Commons Attribution 4.0 International License.

Read Full License 


\section{Abstract}

Background: The combination of transcatheter arterial chemoembolization (TACE) plus sorafenib prolonged progression-free survival (PFS) and overall survival (OS) than sorafenib or TACE monotherapy for patients with hepatocellular carcinoma (HCC). This study assessed the efficacy and safety of radiotherapy (RT) plus monoclonal antibody against programmed cell death 1 (anti-PD1) versus TACE plus sorafenib for patients with advanced HCC.

Methods: Patients with advanced HCC who treated with RT plus anti-PD1 and TACE plus sorafenib were enrolled. Objective response rate (ORR), PFS, disease control rate (DCR) and OS were calculated to assess the antitumor response and the treatment-related adverse events to the safety.

Results: Between January 2018 to March 2021, 37 patients underwent RT plus anti-PD1 and 41 patients underwent TACE plus sorafenib. The baseline characteristics between the two groups were comparable. The ORR and DCR were significantly higher in the RT+PD1 group than the TACE plus sorafenib group according to RECIST 1.1 (54.05\% vs $12.20 \%, \mathrm{P}<0.001 ; 70.27 \%$ vs $46.37 \%, \mathrm{P}=0.041$; respectively) and according to $\mathrm{mRECIST}$ ( $56.76 \%$ vs $31.71 \%, \mathrm{P}=0.039 ; 70.27 \%$ vs $46.37 \%, \mathrm{P}=0.041$; respectively). RT plus anti-PD1 provided significantly better PFS (HR, $0.51 ; 95 \% \mathrm{Cl} 0.30-0.86 ; \mathrm{p}=0.017)$ than TACE plus sorafenib. Moreover, patients with RT plus anti-PD1 had significantly higher 3-, 6-, and 9-month OS rates than those with TACE plus sorafenib( $97.3 \%$ vs $92.30 \%, \mathrm{P}<0.001 ; 91.89 \%$ vs $68.60 \%, \mathrm{P}<0.001 ; 75.5 \%$ vs $60.60 \%, P<0.001$; respectively). The median OS was more favorable 17.4 months for the RT+PD1 group and 11.9 months for the TACE plus sorafenib group. No treatment-related death was observed. Grade 3 or more treatment-related adverse events (TRAEs) occurred significantly less in patients in the RT+PD1 group than the TACE plus sorafenib group $(29.7 \%$ vs $75.6 \%, p<0.001)$, and all TRAEs were manageable.

Conclusions: In this real-world study, RT plus anti-PD1 showed significantly promising efficacy and manageable safety than TACE plus sorafenib in patients with advanced HCC. Toxicities were manageable, with no unexpected safety signals. The study provides evidence on a new therapeutic method in the treatment of advanced HCC.

\section{Background}

Hepatocellular carcinoma (HCC) is the most common type of liver cancer, which is the 6th most common cancer worldwide and the 3rd leading cause of cancer-related death [1]. Most patients are found at an advanced stage having a poor prognosis [2, 3]. TACE, RT and sorafenib are recommended as standard treatments for the patients with advanced HCC by The National Comprehensive Cancer Network (NCCN) guideline [4]. The new treatment strategies, combination of TACE with sorafenib, is proved to be a welltolerated and feasible treatment $[5,6]$. The combination of TACE and sorafenib prolonged progressionfree and overall survival times than sorafenib or TACE monotherapy, and was indicated to be a promising treatment in patients with unresectable HCC [7-9]. 
With the rapid improvement of RT technology and equipment, the curative effect of RT for HCC has been significantly improved in recent years. Hypofractionated RT has also been shown to be effective in patients with HCC through excellent local control, downstaging, conversion from unresectable to resectable status, and treatments of unresectable HCC with vessel invasion or multiple intrahepatic metastases [10-12].

The anti-PD1 has emerged to play a promising role in the treatment of HCC over the last few years [13]. RT can undergo a so-called immunogenic death inducing in situ vaccination and render the tumor microenvironment conducive to effector T-cell 4 recruitment and function to produce a synergistic antitumor immunity with anti-PD1 for durable disease control [14]. Early clinical trials combining RT with antiPD1 showed clinical activity in several cancers including non-small cell lung cancer [15], malignant pigmented tumor [16], and pancreatic adenocarcinoma [17]. A case report including 5 cases showed the impressive tumor control in patients with advanced HCC treated by the combination of RT and anti-PD1 [18].

To the best of our knowledge, there has been rare study to directly compare the efficacy and safety of anti-PD1 plus RT versus other strategies in the treatment of advanced HCC. Therefore, we performed this real-world study to evaluate the efficacy and safety of anti-PD1 plus RT versus TACE plus sorafenib in the treatment of advanced HCC, and seek a new approach for the treatment of advanced HCC.

\section{Methods}

\section{Study design and patients}

This is a real-world study that was conducted at Guangxi Medical University Cancer Hospital, Nanning, China. Consecutive patients were identified via the electronic medical records from January 2018 to March 2021. Of these patients, 99 patients with advanced HCC were screened and 78 patients were included based on the following chief eligibility criteria: (a) patients with advanced HCC not fit for radical cures such as hepatic resection or local ablation and Barcelona Clinic Liver Cancer (BCLC) B or C stage, (b) diagnosed according to histopathology, the European Association for the Study of the Liver (EASL) criteria [19] or American Association for the Study of Liver Diseases (AASLD) [20], (c) received RT plus at least one cycle synchronous, or sequential anti-PD1 or TACE plus synchronous or sequential oral sorafenib, (d) had at least one measurable lesion based on Response Evaluation Criteria in Solid Tumors (RECIST) 1.1 [21] and an Eastern Cooperative Oncology Group (ECOG) performance status 0 or 1, (e) aged between 18 and 70 years, (f) had Child-Pugh class A or B liver function, (g) an observation period of $\geq 1$ months. The key exclusion criteria were patients combined intrahepatic cholangiocarcinoma, with incomplete medical information or lost to follow-up, treatment duration of sorafenib less than 1 month. A total of 78 patients were enrolled in this study with 37 in the RT + PD1 group and 41 in the TACE + sorafinib group.

The study was conducted with approval from the institutional ethics committee of Guangxi Medical University Tumor Hospital. The patient's informed consent was obtained from all participants. 


\section{Anti-PD1 therapy}

In the RT + PD1 group, anti-PD1 included pembrolizumab (Merck Sharp \& Dohme Co., Inc.), camrelizumab (Jiangsu HengRui Medicine Co., Ltd.), toripalimab (Shanghai Junshi Biosciences Co., Ltd), tislelizumab (BeiGene), and sintilimab (Innovent Biologics [Suzhou] Co. Ltd.). Dose, method of injection and duration of anti-PD1 were according to the manufacturers instructions. The anti-PD1 were recommended to be used continually every 2 or 3 weeks until disease progression, or intolerable toxicity.

\section{Hypofractionated intensity modulated radiotherapy}

All patients treated with RT + PD1 underwent enhanced CT scan at 2.5-5mm slice thickness for hypofractionated intensity modulated radiotherapy (hypo-IMRT) planning. Gross tumor volume (GTV) was defined as tumor focus that was visualized on contrast imaging. The clinical target volume (CTV) was defined as GTV plus a margin of $4 \sim 5 \mathrm{~mm}$ as described before [22]. The target volumes and organs at risk (OARs) were contour in the MIM 6.8 system (MIM, USA). Whenever conditions permitted, CTpositron emission tomography (PET-CT) fusion and CT-magnetic resonance imaging (MRI) fusion were performed. For patients with multiple metastases, 1-5 lesions were chosen for hypo-IMRT at the discretion of the radiation oncologists. The planned target volume (PTV) was defined as GTV or CTV plus asymmetrical dilation of $1 \mathrm{~cm}$ in craniocaudal direction and $5 \mathrm{~mm}$ in axial direction to set uncertainty and respiratory movement. The hypo-IMRT plans were designed using the Monaco treatment planning system (TPS) (version 5.1) or Pinnacle 3 system (Philips, Netherlands), and performed by volumetric-modulated arc therapy (VMAT) or intensity modulated radiotherapy (IMRT). The RT was delivered daily over five fractions per week, using a $6 \mathrm{MV}$ X-ray linear accelerator (ELEKTA Versa-HD). Cone-beam computed tomographic (CBCT) images were used to correct the positions.

\section{Sorafenib therapy}

In the TACE + sorafenib group, patients treated with sorafenib were prescribed with two tablets of sorafenib (200 mg tablet) twice daily ( $800 \mathrm{mg} /$ day). If unacceptable treatment-related toxicity or disease progression occurs, dose reduction and treatment termination are allowed.

\section{Transarterial chemoembolization}

TACE was performed as described before [23]. Briefly, all patients in the TACE + sorafenib group underwent selective arteriography of the hepatic artery to locate the tumors and the percutaneous femoral artery was punctured using the Seldinger technique. The combination of local chemotherapy drug solution (cisplatin $80 \mathrm{mg} / \mathrm{m} 2$ or pirarubicin 60-80 mg) and drug carriers (lipiodol or ethiodized poppy seed oil $5-15 \mathrm{~mL}$ ) was introduced into the tumor. And blank CalliSpheres $\mathrm{R}$ microspheres was used to embolize the feeding artery of tumors Complete embolization of the tumor supplying artery with no tumor staining observed by angiography at the end of procedure was defined as a technical success. TACE was performed 1 to 2 months before or after sorafenib and repeated one to six times (median,3) at 3-6-week intervals if the patient could tolerate and consent of further treatment. 


\section{Evaluation of efficacy and safety}

The on-study date was defined as the day of acceptance of informed consent of sorafinib or hypo-IMRT planning. All TRAEs were recorded from on-study date until 30 days after last anti-PD1 injection or sorafinib, last follow-up as appropriate according to the Common Terminology Criteria for Adverse Events of the National Cancer Institute v5.0 (CTCAE 5.0). To evaluate tumour responses, RECIST 1.1 and modified Response Evaluation Criteria in Solid Tumors (mRECIST) [24] were used, respectively. The OS was defined as the on-study date to death from any cause. PFS was defined as the on-study date until disease progression or death. the ORR was defined as the proportion of patients with complete response (CR) or partial response (PR). Disease control was defined as the sum rate of $C R+P R+$ stable disease (SD). All patients received follow-up visits every month for progression and survival status.

\section{Statistical analysis}

The data of all patients was collected through electronic medical records and enter the data into Research Electronic Data Capture (REDCap) [25]. Through the normal distribution test by visualization on a histogram and with Kolmogorov-Smirnov tests, the continuous variables conforming to the normal distribution were expressed as the mean \pm standard deviation and visualized on a histogram and with Kolmogorov-Smirnov tests. The categorical variables were expressed as number and percentage. The ttest was used for the continuous variables conforming to normal distributions, and the Pearson's chisquare tests was used for categorical variables. OS and PFS were calculated and median OS and PFS were estimated for both groups by using Kaplan-Meier methods with the values compared using the Breslow generalized Wilcoxon test [26]. $\mathrm{HR}$ and $\mathrm{Cl}$ were estimated using univariate Cox proportional risk model, and statistical analysis and forest mapping were performed using R 4.0.5 for the subgroups. All statistical tests were two tailed. IBM SPSS software (ver. 26.0 SPSS Inc., Chicago, IL, USA) was used for the statistical analysis unless otherwise indicated.

\section{Results}

\section{Patients}

Of the 78 patients with advanced HCC enrolled, 37 (47.4\%) were in the RT+PD1 group and 41 (52.6\%) were in the TACE + sorafenib group (Fig. 1). The baseline characteristics of these patients were summarized and comparable between the two groups (Table 1). There were no significant differences in the distribution of gender, age, bodyweight, chronic hepatitis B/C virus infection, liver cirrhosis, alpha fetoprotein, albumin-bilirubin (ALBI) grade, maximum tumor diameter, macrovascular invasion, BCLC stage, hemoglobin, platelet count, white blood cell, and prior therapy between the two groups (Table 1). In the RT+PD1 group, 31 patients (83.78\%) had underlying diseases of chronic hepatitis $B$ virus infection (HBV), 18 (48.65\%) with the alpha-fetoprotein (AFP) level $\geq 400,16$ (43.24\%) with the macrovascular invasion (MVI), 8 (21.62\%) had HCC with the largest diameter $\geq 10 \mathrm{~cm}$, and $33(89.19 \%)$ were categorized as BCLC stage C. And in the TACE plus sorafenib group, 31 patients (73.80\%) have HBV, 19 (45.24\%) with 
the alpha-fetoprotein (AFP) level $\geq 400,24$ (57.14\%) with MVI, 13 (30.95\%) had HCC with the largest diameter $\geq 10 \mathrm{~cm}$, and $32(76.19 \%)$ were categorized as BCLC stage C. Patients with extrahepatic metastasis were more prevalent in the RT+PD1 group than in the TACE + sorafenib group $(59.5 \%$ vs. $35.7 \%$, respectively, $p=0.044)$, while Albumin $(<35 \mathrm{~g} / \mathrm{L})$ were more common in the TACE plus sorafenib group than in the TACE plus RT group ( $76.2 \%$ vs $46.0 \%$, respectively; $p=0.010)$. 
Table 1

Patient baseline demographic and clinical characteristics.

\begin{tabular}{|c|c|c|c|}
\hline \multirow[t]{3}{*}{ Variables } & \multirow[t]{3}{*}{ RT+PD1, N=37 (\%) } & \multirow{3}{*}{$\begin{array}{l}\text { TACE + Sorafenib, } \\
\mathrm{N}=41(\%)\end{array}$} & \multirow{3}{*}{$\begin{array}{l}\text { Pvalue } \\
\text { RT+PD1 vs } \\
\text { TACE + sorafenib }\end{array}$} \\
\hline & & & \\
\hline & & & \\
\hline Gender, male & $35(94.60 \%)$ & $37(90.24 \%)$ & 0.678 \\
\hline Age, year & $54.16 \pm 10.68$ & $51.71 \pm 9.571$ & 0.288 \\
\hline Bodyweight, kg & $63.58 \pm 9.96$ & $65.06 \pm 10.84$ & 0.534 \\
\hline Hepatitis B, present & $31(83.78 \%)$ & $30(73.17 \%)$ & 0.286 \\
\hline Hepatitis C, present & $1(2.70 \%)$ & $5(12.2 \%)$ & 0.204 \\
\hline Liver cirrhosis, present & $17(45.95 \%)$ & $26(63.41 \%)$ & 0.171 \\
\hline Alpha fetoprotein, $\geq 400 \mathrm{ng} / \mathrm{ml}$ & $18(48.65 \%)$ & $18(43.90 \%)$ & 0.820 \\
\hline ALBI grade, $\leq 2$ & $36(97.3 \%)$ & $38(93.7 \%)$ & 0.617 \\
\hline blood bilirubin, $₫ 21$ umol/L & $9(24.32 \%)$ & $14(34.15 \%)$ & 0.457 \\
\hline Albumin, $<35 \mathrm{~g} / \mathrm{L}$ & $17(45.95 \%)$ & $31(75.61 \%)$ & 0.010 \\
\hline hemoglobin, <131g/L & $22(59.46 \%)$ & $19(46.34 \%)$ & 0.266 \\
\hline Platelet count, $<100 * 10^{9 /} \mathrm{L}$ & $7(18.92 \%)$ & $6(14.63 \%)$ & 0.763 \\
\hline white blood cell, $<3.97 * 10^{9 / L}$ & $7(18.92 \%)$ & $4(9.76 \%)$ & 0.333 \\
\hline Maximum tumor diameter, $\mathrm{cm}$ & 7.30(4.75-8.65) & $6.20(4.15-10.80)$ & 0.860 \\
\hline Maximum tumor diameter, $\geq 10 \mathrm{~cm}$ & $8(21.62 \%)$ & $13(31.71 \%)$ & 0.444 \\
\hline Macrovascular invasion, present & $16(43.24 \%)$ & $24(58.54 \%)$ & 0.257 \\
\hline Extrahepatic metastasis, present & $22(59.46 \%)$ & $14(34.15 \%)$ & 0.040 \\
\hline BCLC stage & & & 0.147 \\
\hline B & $4(10.81 \%)$ & $10(24.39 \%)$ & \\
\hline C & $33(89.19 \%)$ & $31(75.61 \%)$ & \\
\hline \multicolumn{4}{|l|}{ Prior therapy } \\
\hline TACE, present & $11(29.73 \%)$ & $10(24.39 \%)$ & 0.619 \\
\hline
\end{tabular}

Data are mean \pm standard deviation, median (IQR) or $\mathrm{N}(\%)$.

RT, radiotherapy; PD1, the monoclonal antibody against programmed cell death 1;TACE, transcatheter arterial chemoembolization; ALBI, albumin-bilirubin; BCLC, Barcelona Clinic Liver Cancer. 


\begin{tabular}{|llll|}
\hline Variables & RT+PD1, N=37 (\%) & TACE + Sorafenib, & Pvalue \\
\cline { 3 - 3 } & & N=41(\%) & RT+PD1 vs \\
& & TACE + sorafenib \\
\hline Hepatectomy, present & $16(43.24 \%)$ & $14(34.15 \%)$ & 0.487 \\
\hline Systemic therapy, present & $6(16.22 \%)$ & $6(14.63 \%)$ & 1.000 \\
\hline Data are mean \pm standard deviation, median (IQR) or N (\%). & \\
\hline $\begin{array}{l}\text { RT, radiotherapy; PD1, the monoclonal antibody against programmed cell death 1;TACE, transcatheter } \\
\text { arterial chemoembolization; ALBl, albumin-bilirubin; BCLC, Barcelona Clinic Liver Cancer. }\end{array}$ \\
\hline
\end{tabular}

In the RT+PD1 group, patients received median 5 (1-20) cycles anti-pd1 including 32 patients with camrelizumab (SHR-1210, Jiangsu HengRui Medicine Co., Ltd.) [27], 3 with sintilimab (IBI308, Innovent Biologics [Suzhou] Co. Ltd.) [28], 2 with tislelizumab (BGB-A317, BeiGene) [29]. All patients with much more lesions than hypo-IMRT targets received hypo-IMRT median dose 40 Gy (rang 30-60 Gy) with median 4 Gy per fractions (rang 3-5 Gy per fractions). The median duration of sorafinib therapy was 6.5 (rang 1-20) months and the median number of TACE sessions was 2 (rang 1-6) in the TACE + sorafenib group.

At data cutoff, 14 (37.8\%) patients had received subsequent therapy including 1 received TACE plus hepatectomy, 2 received regorafenib, 2 received sorafinib, 2 received lenvatinib, 3 received TACE, and 4 received apatinib in the RT+PD1 group after disease progression. As to the TACE + sorafenib group, 16 $(39.0 \%)$ patients had received subsequent therapy including one received radiofrequency ablation plus anti-PD1, 1 received hepatectomy plus anti-PD1, 1 received lenvatinib, 1 received lenvatinib plus anti-PD1, 1 received apatinib, 1 received regorafenib plus anti-PD1, 2 received hepatectomy, and 10 received antiPD1.

\section{Radiologic response after treatment}

The median follow-up of the patients in the RT+PD1 group was 13.4 months (95\% $\mathrm{Cl} 12.1-14.6$ ), and in the TACE plus sorafenib group was 16.6 months $(95 \% \mathrm{Cl} 14.9-18.3), p=0.034$. The tumor responses are presented in Table 2. the PR rate, ORR and DCR were significantly higher in the RT+PD1 group than the TACE plus sorafenib group according to RECIST 1.1 (54.05\% vs $12.20 \%, \mathrm{P}<0.001 ; 54.05 \%$ vs $12.20 \%$, $\mathrm{P}<$ $0.001 ; 70.27 \%$ vs $46.34 \%, \mathrm{P}=0.041$; respectively) and according to mRECIST $(56.76 \%$ vs $31.71 \%, \mathrm{P}=$ $0.039 ; 54.05 \%$ vs $12.20 \%, P<0.001 ; 70.27 \%$ vs $46.34 \%, P=0.041$; respectively). Best percent change from baseline in sum of longest diameters for target lesions per patient in the two groups is shown in the waterfall plot (Fig. 2), where Fig. A, B, C, and D represent the results in the RT+PD1 group based on RECIST 1.1, the TACE plus sorafenib group based on RECIST 1.1, the RT+PD1 group based on mRECIST, and the TACE plus sorafenib group based on mRECIST, respectively. 
Table 2

Summary of efficacy outcomes.

\begin{tabular}{|c|c|c|c|}
\hline Variables & $\begin{array}{l}\text { RT+PD1, N=37 } \\
\text { (\%) }\end{array}$ & $\begin{array}{l}\text { TACE + Sorafenib, } \\
\mathrm{N}=41(\%)\end{array}$ & $\begin{array}{l}\mathrm{P} \\
\text { value }\end{array}$ \\
\hline \multicolumn{4}{|l|}{ According to RECIST 1.1} \\
\hline Confirmed objective response & $20(54.05 \%)$ & $5(12.20 \%)$ & $\begin{array}{l}< \\
0.001\end{array}$ \\
\hline Time to response, months, (IQR) & $1.80(1.57-2.00)$ & $2.87(1.407-6.86)$ & 0.467 \\
\hline Disease control & $26(70.27 \%)$ & $19(46.34 \%)$ & 0.041 \\
\hline \multicolumn{4}{|l|}{ Best overall response } \\
\hline CR & $0(0)$ & $0(0)$ & 1.000 \\
\hline PR & $20(54.05 \%)$ & $5(12.20 \%)$ & $\begin{array}{l}<.001 \\
0.001\end{array}$ \\
\hline SD & $6(16.22 \%)$ & $14(34.15 \%)$ & 0.118 \\
\hline PD & $11(29.73 \%)$ & $22(53.66 \%)$ & 0.041 \\
\hline $\begin{array}{l}\text { Progression-free survival, months, median, } \\
(95 \% \mathrm{Cl})\end{array}$ & $5.86(3.19-8.53)$ & $3.70(2.60-4.80)$ & 0.017 \\
\hline \multicolumn{4}{|l|}{ According to mRECIST } \\
\hline Confirmed objective response & $21(56.76 \%)$ & $13(31.71 \%)$ & 0.039 \\
\hline Time to response, months, (IQR) & $1.85(1.60-2.98)$ & $1.47(0.80-2.60)$ & 0.061 \\
\hline Disease control & $26(70.27 \%)$ & $19(46.34 \%)$ & 0.041 \\
\hline \multicolumn{4}{|l|}{ Best overall response } \\
\hline $\mathrm{CR}$ & $1(2.70 \%)$ & $1(2.44 \%)$ & 1.000 \\
\hline PR & 20 (54.05\%) & $12(29.27 \%)$ & 0.038 \\
\hline SD & $5(13.51 \%)$ & $6(14.63 \%)$ & 0.774 \\
\hline PD & $11(29.73 \%)$ & $22(53.66 \%)$ & 0.041 \\
\hline $\begin{array}{l}\text { Progression-free survival, months, median, } \\
(95 \% \mathrm{Cl})\end{array}$ & $5.86(3.19-8.53)$ & $3.70(2.60-4.80)$ & 0.019 \\
\hline
\end{tabular}

Data are $\mathrm{n}(\% ; 95 \% \mathrm{Cl})$, unless indicated.

RT, radiotherapy; PD1, the monoclonal antibody against programmed cell death $1 ; T A C E$, transcatheter arterial chemoembolization; RECIST 1.1, Response Evaluation Criteria in Solid Tumors 1.1; mRECIST, modified Response Evaluation Criteria in Solid Tumors; CR, Complete response; PR, Partial response; SD, Stable disease; PD, Progressive disease. 


\begin{tabular}{|c|c|c|c|}
\hline Variables & $\begin{array}{l}\text { RT+PD1, N=37 } \\
\text { (\%) }\end{array}$ & $\begin{array}{l}\text { TACE + Sorafenib, } \\
\mathrm{N}=41(\%)\end{array}$ & $\begin{array}{l}P \\
\text { value }\end{array}$ \\
\hline 3-month, \% & $97.30 \%$ & $92.30 \%$ & $\begin{array}{l}< \\
0.001\end{array}$ \\
\hline 6-month, \% & $91.90 \%$ & $68.60 \%$ & $\begin{array}{l}< \\
0.001\end{array}$ \\
\hline 9-month, \% & $75.50 \%$ & $60.60 \%$ & $\begin{array}{l}< \\
0.001\end{array}$ \\
\hline 12-month, \% & $52.20 \%$ & $47.5 \%$ & 0.061 \\
\hline Overall survival, months, median, $(95 \% \mathrm{Cl})$ & $\begin{array}{l}11.90(6.35- \\
17.45)\end{array}$ & $17.40(8.69-26.11)$ & 0.146 \\
\hline \multicolumn{4}{|l|}{ Data are $\mathrm{n}(\% ; 95 \% \mathrm{Cl})$, unless indicated. } \\
\hline \multicolumn{4}{|c|}{$\begin{array}{l}\text { RT, radiotherapy; PD1, the monoclonal antibody against programmed cell death } 1 ; \text { TACE, transcatheter } \\
\text { arterial chemoembolization; RECIST 1.1, Response Evaluation Criteria in Solid Tumors } 1.1 ; \mathrm{mRECIST} \text {, } \\
\text { modified Response Evaluation Criteria in Solid Tumors; CR, Complete response; PR, Partial response; } \\
\text { SD, Stable disease; PD, Progressive disease. }\end{array}$} \\
\hline
\end{tabular}

\section{Progression-free survival analysis}

Progression of the disease was observed in 37 of the patients (47.4\%) who underwent RT+PD1 and 41 of the patients who underwent TACE plus sorafenib group (52.8\%) during follow-up. Patients in the PD1+RT group had significant better PFS compared with those in the TACE + sorafenib group (5.86 vs 3.70 months; HR, 0.51; 95\% Cl 0.30-0.86; $\mathrm{p}=0.017$; Table 2 and Fig. 3). The median PFS in the PD1+RT group had significant longer than TACE + sorafenib group across subgroups based on age $\geq 53$, infection of hepatitis $B(\mathrm{HBV})$, the maximum tumor diameter $<10 \mathrm{~cm}, \mathrm{MVI}$, without extrahepatic metastasis, BCLC stage $C$, no prior TACE, prior hepatectomy, and no prior systemic therapy $(p=0.01,0.04,0.031,0.024$, $0.049,0.0067,0.044,0.024$ and 0.016 ; respectively, Fig. $4 \mathrm{~A})$. The effect of the two groups on median PFS was consistent across subgroups based on other baseline characteristics (Fig. 4A).

\section{Overall survival analysis}

Among the 37 patients in the RT+PD1 group, 18 are still alive, 18 have died, and 1 is lost to follow-up by the end of this study. Among the 41 patients who received TACE plus sorafenib, 13 are still alive, 25 have died, and 3 are lost to follow-up. The 3-, 6-, and 9-month OS rates were significant better in the RT+PD1 group than that in the TACE plus sorafenib $(97.3 \%$ vs $92.30 \%, \mathrm{P}<0.001 ; 91.89 \%$ vs $68.60 \%, \mathrm{P}<0.001$; $75.5 \%$ vs $60.60 \%, P<0.001$; respectively, Table 2 ). The median OS was 17.4 months for the RT+PD1 group and 11.9 months for the TACE plus sorafenib group (HR: $0.70,95 \% \mathrm{Cl}, 0.38-1.30, \mathrm{P}=0.146$; Table 2 and Fig. 3B). There were no significant differences for the median OS in the distribution of the two groups, but a trend toward a more favorable outcome in the RT+PD1 group. 
Patients in the RT+PD1 group had statistically significant better OS than those in the TACE plus sorafenib group with AFP level $\geq 400 \mathrm{ng} / \mathrm{ml}$, BCLC stage $\mathrm{C}$ and MVI (11.8 vs 9.7 months, $\mathrm{P}=0.026 ; 11.8$ vs 9.7 months, $\mathrm{P}=0.031 ; 11.8$ vs 9.7 months, $\mathrm{P}=0.026$; respectively, Fig. 5). Although, there had not significant better OS with BCLC stage $\mathrm{C}$ and MVI using univariate Cox proportional risk model and R 4.0.5. The effect of the two groups on median OS was consistent across subgroups based on other baseline characteristics (Fig. 4B).

\section{Safety profile}

The TRAEs, which occurred in $\geq 10 \%$ of patients, are summarized in Table 3 . No deaths were attributed to TRAEs in this study. The TRAEs in the RT+PD1 group show less than in the TACE plus sorafenib group, including increased AST (54.05\% vs 92.68\%, p < 0.001), increased AST $(51.35 \%$ vs $80.49 \%, p=0.008)$, hand-foot skin reaction (HFSR) ( $0 \%$ vs $53.66 \%, p<0.001)$. Decreased hemoglobin was more frequent in the RT+PD1 group than in the TACE plus sorafenib group $(89.19 \%$ vs $68.29 \%, p=0.031)$. There were no differences in regard to other TRAEs such as decreased albumin, decreased white blood cell, increased blood bilirubin and decreased platelet count. Grade 3 or more TRAEs occurred significantly less in patients in the RT+PD1 group than the TACE plus sorafenib group $(29.7 \%$ vs $75.6 \%, p<0.001)$. The major grade 3 or more TRAEs were observed significantly milder in the RT+PD1 group than in the TACE plus sorafenib group, including increased aspartate aminotransferase (AST) (58.54\%\% vs 5.41\%, $p=0.000)$, increased alanine aminotransferase (ALT) (36.59\% vs 5.41\%, $p=0.001)$ and hand-foot skin reaction (14.63\% vs $0 \%, p=0.027)$. The two groups did not differ in terms of other grade 3 or more TRAEs including decreased hemoglobin, decreased albumin, decreased white blood cell, increased blood bilirubin and decreased platelet count.

Table 3. Treatment-related adverse events based on CTCAE 5.0. 


\begin{tabular}{|c|c|c|c|c|c|c|}
\hline \multirow[t]{2}{*}{ Adverse events } & \multicolumn{2}{|c|}{$\mathrm{RT}+\mathrm{PD} 1, \mathrm{n}=37(\% \rrbracket$} & \multicolumn{2}{|c|}{ 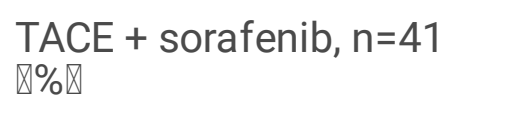 } & \multicolumn{2}{|l|}{$P$ value } \\
\hline & $\begin{array}{l}\text { Any grade } \\
(\%)\end{array}$ & $\begin{array}{l}\text { Grade 3-4 } \\
(\%)\end{array}$ & $\begin{array}{l}\text { Any grade } \\
(\%)\end{array}$ & $\begin{array}{l}\text { Grade 3-4 } \\
(\%)\end{array}$ & $\begin{array}{l}\text { Any } \\
\text { grade }\end{array}$ & $\begin{array}{l}\text { Grade } \\
3-4\end{array}$ \\
\hline Increased AST & $20(54.05 \%)$ & $2 \rrbracket 5.41 \% \rrbracket$ & $38(92.68 \%)$ & $\begin{array}{l}24 \\
₫ 58.54 \% \rrbracket\end{array}$ & $<0.001$ & $<0.001$ \\
\hline Increased ALT & $19(51.35 \%)$ & $2 \rrbracket 5.41 \% \rrbracket$ & $33(80.49 \%)$ & $\begin{array}{l}15 \\
\text { 邓36.59\%区 }\end{array}$ & 0.008 & 0.001 \\
\hline $\begin{array}{l}\text { Hand-foot skin } \\
\text { reaction }\end{array}$ & $0(0)$ & $0(0)$ & $22(53.66 \%)$ & $\begin{array}{l}6 \\
\square 14.63 \% \bigotimes\end{array}$ & $<0.001$ & 0.027 \\
\hline $\begin{array}{l}\text { Decreased } \\
\text { hemoglobin }\end{array}$ & 33(89.19\%) & $5 \rrbracket 13.51 \% \rrbracket$ & $28(68.29 \%)$ & $2 \rrbracket 4.88 \% \bigotimes$ & 0.031 & 0.247 \\
\hline Decreased albumin & $30(81.08 \%)$ & $0 \rrbracket 0 \rrbracket$ & $37(90.24 \%)$ & $1 \rrbracket 2.44 \% \bigotimes$ & 0.333 & 1.000 \\
\hline $\begin{array}{l}\text { Decreased white } \\
\text { blood cell }\end{array}$ & $28(75.68 \%)$ & $3 \llbracket 8.11 \% \rrbracket$ & $12(29.27 \%)$ & $1 \rrbracket 2.44 \% \bigotimes$ & $<0.001$ & 0.341 \\
\hline $\begin{array}{l}\text { Increased blood } \\
\text { bilirubin }\end{array}$ & $20(54.05 \%)$ & $1 \otimes 2.70 \otimes$ & $16(39.02 \%)$ & $1 \rrbracket 2.44 \% \rrbracket$ & 0.256 & 1.000 \\
\hline $\begin{array}{l}\text { Decreased platelet } \\
\text { count }\end{array}$ & $19(51.35 \%)$ & $8 \rrbracket 21.62 \% \rrbracket$ & $14(34.115 \%)$ & $\begin{array}{l}5 \\
\square 12.20 \% \bigotimes\end{array}$ & 0.169 & 0.364 \\
\hline
\end{tabular}

Data are $\mathrm{n}(\%)$.

CTCAE 5.0, the Common Terminology Criteria for Adverse Events of the National Cancer Institute v5.0; RT, radiotherapy; PD1, the monoclonal antibody against programmed cell death 1;TACE, transcatheter arterial chemoembolization; AST, aspartate aminotransferase; ALT, alanine aminotransferase.

\section{Discussion}

Few studies have investigated the efficacy and safety of combination RT plus anti-PD1 versus TACE plus sorafenib in the treatment of advanced HCC in the real-world study. The present observational study showed significantly better PFS and ORR with combining RT plus anti-PD1 than TACE plus sorafenib in patients with advanced HCC. The TRAEs were acceptable and milder in patients treated with RT plus antiPD1 than with TACE plus sorafenib. Combining anti-PD1 with RT might be a potential beneficial strategy to efficiently and safely treat the patients with advanced HCC.

Sorafenib is recommended as the first-line treatment for the patients with advance HCC [4]. The outcome of patients who received sorafenib in our study are better than previous studies with consistent baseline. Patients with advanced HCC reached the median OS to 6.5 months, DCR to $35.3 \%$, and the ORR to $3.3 \%$ in Asia Pacific patients with sorafenib [30], and 10.4 months, $64 \%, 4 \%$ in patients with sorafenib respectively in ORIENT-32 study [28]. Both treatments of our study achieved better outcome. Similarly, the recent 
studies of combined TACE plus sorafenib have been conducted in patients with unresectable HCC, and combined treatments show an enhanced efficacy as compared to TACE or sorafenib alone $[6,8,9,31]$. In the GIDEON study, the median OS was 12.7 months in prior-TACE group, 9.2 months in non-prior-TACE, 21.6 months in concomitant-TACE, and 9.7 months in non-concomitant-TACE one for patients treated with sorafinib, respectively. the median OS of combined TACE plus sorafenib in our study was 11.9 months $(95 \% \mathrm{Cl}, 6.35-17.45)$ and was similar to the prior-TACE group but shorter than concomitant-TACE group in the GIDEON study. Notably, the tumor stage of BCLC and the rate of chronic HBV present were much higher in our study than the GIDEON study ( $A, 0$ vs $10.5 \% ; B, 24.39 \%$ vs $30.2 \%$, for stage respectively; $73.17 \%$ vs $43.1 \%$ for the rate of $\mathrm{HBV}$ ), meaning more prone to develop progressive disease and have poorer prognoses [32]. These findings suggest that combining TACE plus sorafenib contribute to the overall treatment benefit in patients with HCC.

In our real-word study, the PR rate, ORR and DCR were significantly higher in the RT+PD1 group than the TACE plus sorafenib group according to RECIST 1.1 (54.05\% vs $12.20 \%, \mathrm{P}<0.001 ; 54.05 \%$ vs $12.20 \%, \mathrm{P}<$ $0.001 ; 70.27 \%$ vs $46.34 \%, \mathrm{P}=0.041$; respectively) and according to mRECIST $(56.76 \%$ vs $31.71 \%, \mathrm{P}=$ $0.039 ; 54.05 \%$ vs $12.20 \%, \mathrm{P}<0.001 ; 70.27 \%$ vs $46.34 \%, \mathrm{P}=0.041$; respectively). The ORR was better than in the IMbrave150 trial (27.3\%) [33] and in the REFELECT trial (24.1\%) [34]. Significantly better PFS was showed in this study for patients with advanced HCC with combining RT plus anti-PD1 than TACE plus sorafenib (5.86 vs 3.70 months, HR, $0.51, p=0.017$ ). The 3-, 6-, and 9-month OS rates were significant better in the RT+PD1 group than that in the TACE plus sorafenib group ( $97.3 \%$ vs $92.30 \%, \mathrm{P}<0.001$; $91.89 \%$ vs $68.60 \%, \mathrm{P}<0.001 ; 75.5 \%$ vs $60.60 \%, \mathrm{P}<0.001$; respectively). The 6 -month OS rates was better in the RT+PD1 group than in the IMbrave150 trial (84.8\%) [33]. There is a trend toward a more favorable median OS in the RT+PD1 group than TACE plus sorafenib (17.4 months vs 11.9 months; HR, $0.70,95 \%$ $\mathrm{Cl}, 0.38-1.30)$. It is worth noting that the median follow-up of the patients in the RT+PD1 group was significantly shorter than the TACE plus sorafenib group (13.4 months, $95 \% \mathrm{Cl}, 12.1-14.6$ vs 16.6 months, $95 \% \mathrm{Cl}, 14.9-18.3 ; \mathrm{p}=0.034)$. These may account for no significantly better $\mathrm{OS}$ in the present study. Considering the statistically significant shouter in median follow-up in the RT+PD1 group than that in the TACE plus sorafenib group, the median OS and PFS were estimated for both groups by using KaplanMeier methods with the values compared using the Breslow generalized Wilcoxon test giving more weight to earlier events [35]. These findings suggest that both combining RT plus anti-PD1 and TACE plus sorafenib contribute to the overall treatment benefit in patients with advanced HCC.

Meanwhile, subgroup analysis for PFS and OS was done. The median PFS in the PD1+RT group had significant longer than TACE + sorafenib group across subgroups based on age $\geq 53$, infection of hepatitis $B(\mathrm{HBV})$, the maximum tumor diameter $₫ 10 \mathrm{~cm}, \mathrm{MVl}$, without extrahepatic metastasis, BCLC stage $C$, no prior TACE, prior hepatectomy, and no prior systemic therapy $(p=0.01,0.04,0.031,0.024$, $0.049,0.0067,0.044,0.024$ and 0.016 ; respectively). Patients in the RT+PD1 group had statistically significant better OS than those in the TACE + sorafenib group with AFP level $\geq 400 \mathrm{ng} / \mathrm{ml}$, BCLC stage C and $\mathrm{MVI}$ (11.8 vs 9.7 months, $P=0.026 ; 11.8$ vs 9.7 months, $P=0.031 ; 11.8$ vs 9.7 months, $P=0.026$ ). Although, there had not significant better OS with BCLC stage $C$ and MVI using univariate Cox proportional risk model and R 4.0.5. The shorter follow-up may account for no significantly better OS. 
Together, these findings may help to select patients with advance HCC who would derive an OS and PFS benefit from combining RT plus anti-PD1 and TACE plus sorafenib. However, further investigation and confirmation are needed.

The spectrum, incidence, and severity of adverse events in the TACE plus sorafenib group were consistent with the known safety profile observed in other studies[5, 7-9, 36]. The TRAEs in the RT+PD1 group were less than in the TACE plus sorafenib group, including increased AST ( $54.05 \%$ vs $92.68 \%, p<0.001)$, increased AST $(51.35 \%$ vs $80.49 \%, p=0.008)$, hand-foot skin reaction (HFSR) ( $0 \%$ vs $53.66 \%, p<0.001)$. Grade 3 or more TRAEs occurred significantly less in patients in the RT+PD1 group than the TACE plus sorafenib group $(29.7 \%$ vs $75.6 \%, p<0.001)$. The major grade 3 or more TRAEs were increased AST ( $58.54 \% \%$ vs $5.41 \%, p=0.000)$, increased ALT $(36.59 \%$ vs $5.41 \%, p=0.001)$ and hand-foot skin reaction $(14.63 \%$ vs $0 \%, p=0.027)$.

This study had several limitations. First, the nature of this retrospective design study was the main drawback and vulnerable to other potential biases, such as adherence and different socioeconomic status among patients. Second, the follow-up period was not long enough in the RT + PD1 group and significantly shorter than the TACE plus sorafenib group. However, the follow-up duration was sufficient to evaluate PFS and tumor response in the RT + PD1 group were better than the other group, which are more accurately reflect efficacy than OS [37]. Third, three types of anti-PD1 were used in this study and may also be a confounding factor. There have no clinical studies to compare for anti-PD1 to provide evidence of which one works best. Finally, some adverse events, such as cardiotoxicity, could not obtain in some patients due to the absence of standard hematological tests.

\section{Conclusion}

Treatment with RT plus anti-PD1 was associated with significantly better PFS, PR rate, ORR and DCR outcomes than TACE plus sorafenib in patients with advanced HCC, and better OS than TACE plus sorafenib in patients with BCLC stage $C$, macrovascular invasion, or AFP level $\geq 400 \mathrm{ng} / \mathrm{ml}$. The adverse events treated with RT plus anti-PD1 were acceptable and milder than TACE plus sorafenib. Combining anti-PD1 with RT might be a novel potential beneficial strategy to treat the patients with advanced HCC. Additional follow-up and larger sample sizes are needed to draw more accurate conclusions.

\section{Abbreviations}

TACE, transcatheter arterial chemoembolization; PFS, progression-free survival; OS, overall survival; HCC, hepatocellular carcinoma; RT, radiotherapy; anti-PD1, monoclonal antibody against programmed cell death 1; ORR; objective response rate; DCR, disease control rate; HR, hazard ratio; TRAE, treatment-related adverse events; NCCN, The National Comprehensive Cancer Network; BCLC, Barcelona Clinic Liver Cancer; EASL, European Association for the Study of the Liver; AASLD, American Association for the Study of Liver Diseases; RECIST, Response Evaluation Criteria in Solid Tumors; ECOG, Eastern Cooperative Oncology Group; hypo-IMRT, hypofractionated intensity modulated radiotherapy; CTCAE 5.0, Common 
Terminology Criteria for Adverse Events of the National Cancer Institute v5.0; mRECIST, modified Response Evaluation Criteria in Solid Tumors; CR, complete response; PR, partial response; SD, stable disease; $\mathrm{ALBI}$, albumin-bilirubin; MVI, macrovascular invasion; $\mathrm{HBV}$, chronic hepatitis $\mathrm{B}$ virus infection; AFP, alpha-fetoprotein; ALT, alanine aminotransferase; AST, aspartate aminotransferase.

\section{Declarations}

\section{Ethics approval and consent to participate:}

All the procedures were carried out in accordance with the Helsinki Declaration of 1975. This study was approved by the institutional review board of Guangxi Medical University Cancer Hospital (number LW20211104 ). Patient consent was waived due to the retrospective nature of the study.

\section{Consent for publication:}

Not applicable.

\section{Data availability:}

The data underlying this article will be shared on reasonable request to the corresponding author.

\section{Competing interests:}

The authors have no conflict of interest to declare that are relevant to the content of this article.

\section{Funding:}

This study was funded by the Development and Application Project for the Appropriate Technology of Health of Guangxi Province (No. S2019039), the Self-Raised Scientific Research Fund of the Ministry of Health of Guangxi Province (Z20201371) , and the Youth Program of Scientific Research Foundation of Guangxi Medical University Cancer Hospital (2021-10).

\section{Author contributions:}

XBD conceived the study; all authors participated in the acquisition of the data; LJX and DWX analyzed data; LJX, HST and LXF assessed tumor response; LJX drafted and revised the manuscript. All authors read and approved the final manuscript.

\section{Acknowledgements:}

We wish to thank all patients involved in this study and our colleagues at Guangxi Medical University Cancer Hospital.

\section{Author details:}


1. Department of Radiation Oncology, Guangxi Medical University Cancer Hospital, Nanning 530021, China;

2. School of Public Health, Guangxi Medical University, Nanning 530021, China;

3. Department of Hepatobiliary Surgery, Guangxi Medical University Cancer Hospital, Nanning 530021, China.

\section{References}

1. Siegel RL, Miller KD, Jemal A. Cancer statistics, 2020. CA Cancer J Clin. 2020;70(1):7-30.

2. Zhong JH, Peng NF, You XM, Ma L, Xiang X, Wang YY, Gong WF, Wu FX, Xiang BD, Li LQ. Tumor stage and primary treatment of hepatocellular carcinoma at a large tertiary hospital in China: A real-world study. Oncotarget. 2017;8(11):18296-302.

3. Schöniger-Hekele M, Müller C, Kutilek M, Oesterreicher C, Ferenci P, Gangl A. Hepatocellular carcinoma in Central Europe: prognostic features and survival. Gut. 2001;48(1):103-9.

4. Benson AB, D'Angelica MI, Abbott DE, Anaya DA, Anders R, Are C, Bachini M, Borad M, Brown D, Burgoyne A, et al. Hepatobiliary Cancers, Version 2.2021, NCCN Clinical Practice Guidelines in Oncology. J Natl Compr Canc Netw. 2021;19(5):541-65.

5. Chao Y, Chung YH, Han G, Yoon JH, Yang J, Wang J, Shao GL, Kim BI, Lee TY. The combination of transcatheter arterial chemoembolization and sorafenib is well tolerated and effective in Asian patients with hepatocellular carcinoma: final results of the START trial. Int J Cancer. 2015;136(6):1458-67.

6. Geschwind JF, Kudo M, Marrero JA, Venook AP, Chen XP, Bronowicki JP, Dagher L, Furuse J, Ladrón de Guevara L, Papandreou C, et al. TACE Treatment in Patients with Sorafenib-treated Unresectable Hepatocellular Carcinoma in Clinical Practice: Final Analysis of GIDEON. Radiology. 2016;279(2):630-40.

7. Pawlik TM, Reyes DK, Cosgrove D, Kamel IR, Bhagat N, Geschwind JF. Phase II trial of sorafenib combined with concurrent transarterial chemoembolization with drug-eluting beads for hepatocellular carcinoma. J Clin Oncol. 2011;29(30):3960-7.

8. Choi GH, Shim JH, Kim MJ, Ryu MH, Ryoo BY, Kang YK, Shin YM, Kim KM, Lim YS, Lee HC. Sorafenib alone versus sorafenib combined with transarterial chemoembolization for advanced-stage hepatocellular carcinoma: results of propensity score analyses. Radiology. 2013;269(2):603-11.

9. Park JW, Kim YJ, Kim DY, Bae SH, Paik SW, Lee YJ, Kim HY, Lee HC, Han SY, Cheong JY, et al. Sorafenib with or without concurrent transarterial chemoembolization in patients with advanced hepatocellular carcinoma: The phase III STAH trial. J Hepatol. 2019;70(4):684-91.

10. Liang SX, Zhu XD, Lu HJ, Pan CY, Li FX, Huang QF, Wang AY, Chen L, Fu XL, Jiang GL. Hypofractionated three-dimensional conformal radiation therapy for primary liver carcinoma. Cancer. 2005;103(10):2181-8. 
11. Hong TS, Wo JY, Yeap BY, Ben-Josef E, McDonnell El, Blaszkowsky LS, Kwak EL, Allen JN, Clark JW, Goyal L, et al. Multi-Institutional Phase II Study of High-Dose Hypofractionated Proton Beam Therapy in Patients With Localized, Unresectable Hepatocellular Carcinoma and Intrahepatic Cholangiocarcinoma. J Clin Oncol. 2016;34(5):460-8.

12. Choi SH, Seong J. Strategic application of radiotherapy for hepatocellular carcinoma. Clin Mol Hepatol. 2018;24(2):114-34.

13. Rao Q, Li M, Xu W, Pang K, Guo X, Wang D, Liu J, Guo W, Zhang Z. Clinical benefits of PD-1/PD-L1 inhibitors in advanced hepatocellular carcinoma: a systematic review and meta-analysis. Hepatol Int. 2020;14(5):765-75.

14. Herrera FG, Bourhis J, Coukos G. Radiotherapy combination opportunities leveraging immunity for the next oncology practice. CA Cancer J Clin. 2017;67(1):65-85.

15. Shaverdian N, Lisberg AE, Bornazyan K, Veruttipong D, Goldman JW, Formenti SC, Garon EB, Lee P. Previous radiotherapy and the clinical activity and toxicity of pembrolizumab in the treatment of nonsmall-cell lung cancer: a secondary analysis of the KEYNOTE-001 phase 1 trial. The Lancet Oncology. 2017;18(7):895-903.

16. Carron R, Gaudy-Marqueste C, Amatore F, Padovani L, Malissen N, Balossier A, Loundou A, Bonnet N, Muracciole $X$, Régis JM, et al. Stereotactic radiosurgery combined with anti-PD1 for the management of melanoma brain metastases: A retrospective study of safety and efficacy. Eur $\mathrm{J}$ Cancer. 2020;135:52-61.

17. Xie C, Duffy AG, Brar G, Fioravanti S, Mabry-Hrones D, Walker M, Bonilla CM, Wood BJ, Citrin DE, Gil Ramirez EM, et al. Immune Checkpoint Blockade in Combination with Stereotactic Body Radiotherapy in Patients with Metastatic Pancreatic Ductal Adenocarcinoma. Clin Cancer Res. 2020;26(10):2318-26.

18. Chiang CL, Chan ACY, Chiu KWH, Kong FS. Combined Stereotactic Body Radiotherapy and Checkpoint Inhibition in Unresectable Hepatocellular Carcinoma: A Potential Synergistic Treatment Strategy. Front Oncol. 2019;9:1157.

19. EASL Clinical Practice Guidelines. Management of hepatocellular carcinoma. J Hepatol. 2018;69(1):182-236.

20. Marrero JA, Kulik LM, Sirlin CB, Zhu AX, Finn RS, Abecassis MM, Roberts LR, Heimbach JK. Diagnosis, Staging, and Management of Hepatocellular Carcinoma: 2018 Practice Guidance by the American Association for the Study of Liver Diseases. Hepatology. 2018;68(2):723-50.

21. Eisenhauer EA, Therasse P, Bogaerts J, Schwartz LH, Sargent D, Ford R, Dancey J, Arbuck S, Gwyther $\mathrm{S}$, Mooney $\mathrm{M}$, et al. New response evaluation criteria in solid tumours: revised RECIST guideline (version 1.1). Eur J Cancer. 2009;45(2):228-47.

22. Su TS, Li LQ, Liang SX, Xiang BD, Li JX, Ye JZ, Li LQ. A Prospective Study of Liver Regeneration After Radiotherapy Based on a New (Su'S) Target Area Delineation. Front Oncol. 2021;11:680303.

23. Su TS, Li LQ, Meng WW, Wang YD, Chen YT, Li JX, Du YQ, Qu S, Zhao C, Huang DJ, et al. Long-Term Survival Analysis of Transarterial Chemoembolization Plus Radiotherapy vs. Radiotherapy for 
Hepatocellular Carcinoma With Macroscopic Vascular Invasion. Front Oncol. 2020;10:1205.

24. Lencioni R, Llovet JM. Modified RECIST (mRECIST) assessment for hepatocellular carcinoma. Semin Liver Dis. 2010;30(1):52-60.

25. Harris PA, Taylor R, Thielke R, Payne J, Gonzalez N, Conde JG. Research electronic data capture (REDCap)-a metadata-driven methodology and workflow process for providing translational research informatics support. J Biomed Inform. 2009;42(2):377-81.

26. Breslow N. A generalized Kruskal-Wallis test for comparing $\mathrm{K}$ samples subject to unequal patterns of censorship. Biometrika. 1970;57(3):579-94.

27. Qin S, Ren Z, Meng Z, Chen Z, Chai X, Xiong J, Bai Y, Yang L, Zhu H, Fang W, et al. Camrelizumab in patients with previously treated advanced hepatocellular carcinoma: a multicentre, open-label, parallel-group, randomised, phase 2 trial. The Lancet Oncology. 2020;21(4):571-80.

28. Ren Z, Xu J, Bai Y, Xu A, Cang S, Du C, Li Q, Lu Y, Chen Y, Guo Y, et al. Sintilimab plus a bevacizumab biosimilar (IBI305) versus sorafenib in unresectable hepatocellular carcinoma (ORIENT-32): a randomised, open-label, phase 2-3 study. Lancet Oncol. 2021;22(7):977-90.

29. Shen L, Guo J, Zhang Q, Pan H, Yuan Y, Bai Y, Liu T, Zhou Q, Zhao J, Shu Y, et al: Tislelizumab in Chinese patients with advanced solid tumors: an open-label, non-comparative, phase $1 / 2$ study. $J$ Immunother Cancer 2020, 8(1).

30. Cheng A-L, Kang Y-K, Chen Z, Tsao C-J, Qin S, Kim JS, Luo R, Feng J, Ye S, Yang T-S, et al. Efficacy and safety of sorafenib in patients in the Asia-Pacific region with advanced hepatocellular carcinoma: a phase III randomised, double-blind, placebo-controlled trial. The Lancet Oncology. 2009;10(1):25-34.

31. Kudo M, Ueshima K, Ikeda M, Torimura T, Tanabe N, Aikata H, Izumi N, Yamasaki T, Nojiri S, Hino K, et al. Randomised, multicentre prospective trial of transarterial chemoembolisation (TACE) plus sorafenib as compared with TACE alone in patients with hepatocellular carcinoma: TACTICS trial. Gut. 2020;69(8):1492-501.

32. Cantarini MC, Trevisani F, Morselli-Labate AM, Rapaccini G, Farinati F, Del Poggio P, Di Nolfo MA, Benvegnù $L$, Zoli $M$, Borzio $F$, et al. Effect of the etiology of viral cirrhosis on the survival of patients with hepatocellular carcinoma. Am J Gastroenterol. 2006;101(1):91-8.

33. Finn RS, Qin S, Ikeda M, Galle PR, Ducreux M, Kim TY, Kudo M, Breder V, Merle P, Kaseb AO, et al. Atezolizumab plus Bevacizumab in Unresectable Hepatocellular Carcinoma. N Engl J Med. 2020;382(20):1894-905.

34. Kudo M, Finn RS, Qin S, Han K-H, Ikeda K, Piscaglia F, Baron A, Park J-W, Han G, Jassem J, et al. Lenvatinib versus sorafenib in first-line treatment of patients with unresectable hepatocellular carcinoma: a randomised phase 3 non-inferiority trial. The Lancet. 2018;391(10126):1163-73.

35. Martinez RLMC, Naranjo JD. A pretest for choosing between logrank and wilcoxon tests in the twosample problem. METRON. 2010;68(2):111-25.

36. Geschwind JF, Gholam PM, Goldenberg A, Mantry P, Martin RC, Piperdi B, Zigmont E, Imperial J, Babajanyan S, Foreman PK, et al. Use of Transarterial Chemoembolization (TACE) and Sorafenib in 
Patients with Unresectable Hepatocellular Carcinoma: US Regional Analysis of the GIDEON Registry. Liver Cancer. 2016;5(1):37-46.

37. Llovet JM, Villanueva A, Marrero JA, Schwartz M, Meyer T, Galle PR, Lencioni R, Greten TF, Kudo M, Mandrekar SJ, et al: Trial Design and Endpoints in Hepatocellular Carcinoma: AASLD Consensus Conference. Hepatology 2021, 73 Suppl 1:158-191.

\section{Figures}

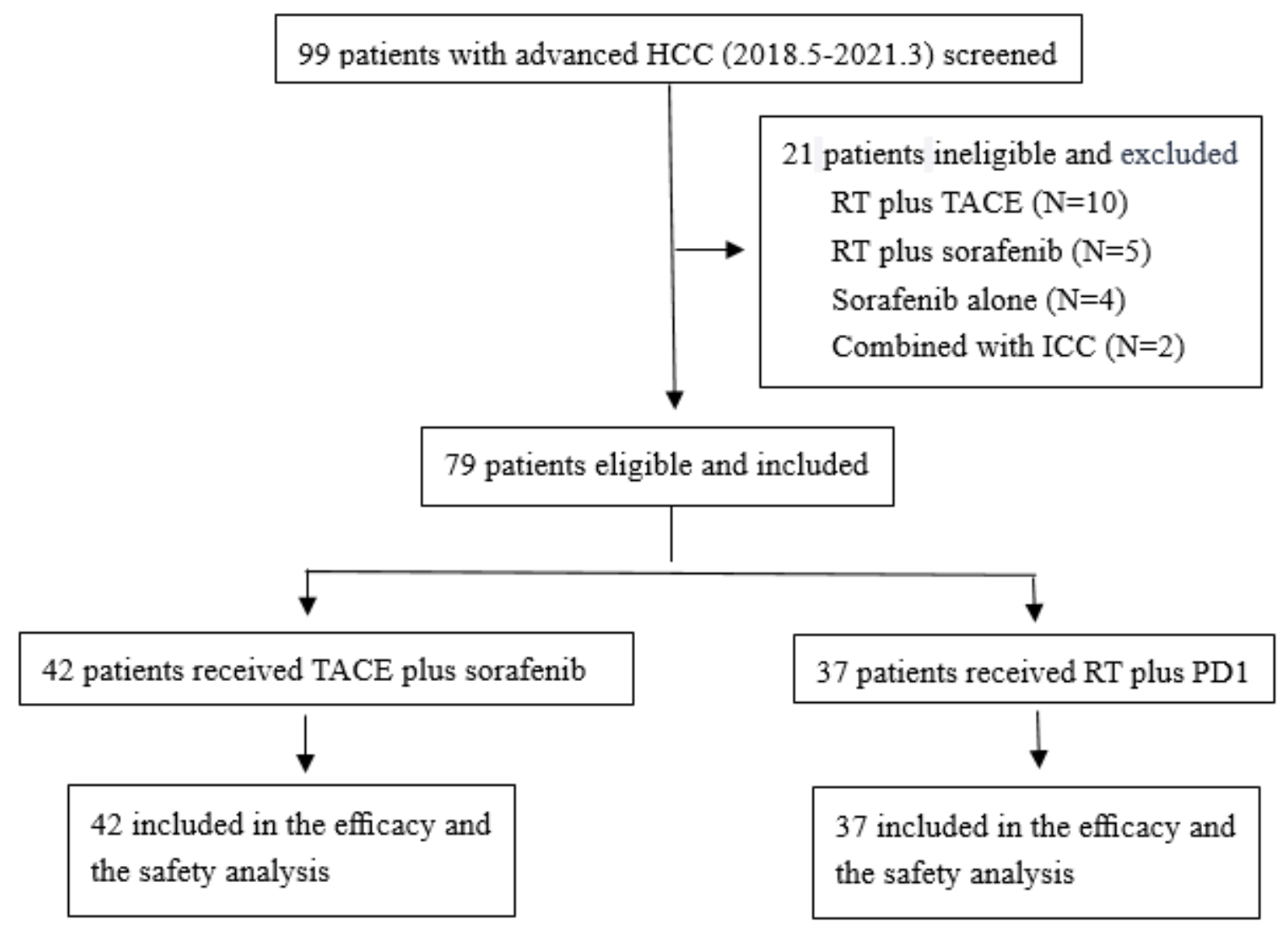

Figure 1

Patient selection flow. HCC, hepatocellular carcinoma; TACE, transcatheter arterial chemoembolization; ICC, intrahepatic cholangiocarcinoma; RT, radiotherapy; PD1, monoclonal antibody against programmed cell death.

\section{Figure 2}

Best percentage change from baseline in sums of diameters of target lesions. (A) In the RT+PD1 group based on RECIST 1.1, (B) In the TACE plus sorafenib group based on RECIST 1.1, (C) In the RT+PD1 group based on mRECIST, (D) In the TACE plus sorafenib group based on mRECIST. RT, radiotherapy; PD1, 
monoclonal antibody against programmed cell death; TACE, transcatheter arterial chemoembolization; RECIST 1.1, Response Evaluation Criteria in Solid Tumors 1.1; mRECIST, modified Response Evaluation Criteria in Solid Tumors; CR, complete response; PR, partial response; SD, stable disease; PD, progressive disease.

\section{Figure 3}

Kaplan-Meier analysis of overall and progression free survival. (A) PFS and (B) OS for all patients. HR, hazard ratio; RT, radiotherapy; PD1, monoclonal antibody against programmed cell death; TACE, transcatheter arterial chemoembolization; OS, overall survival; PFS, progression-free survival.
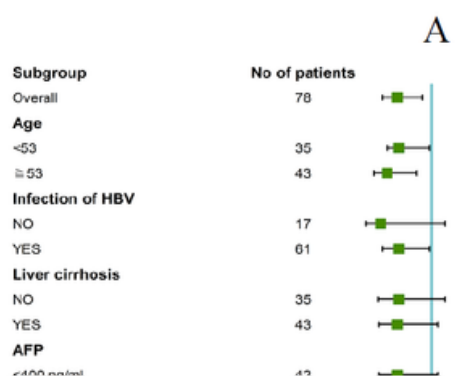

A

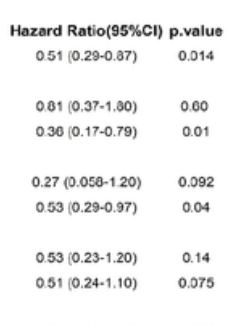

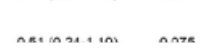

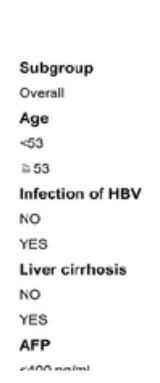

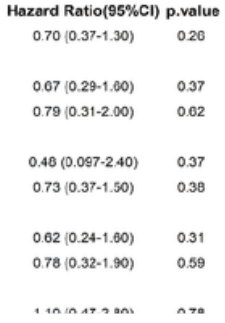

\section{Figure 4}

Forest plot of PFS (A) and OS (B) in subgroups of patients. RT, radiotherapy; PD1, monoclonal antibody against programmed cell death; TACE, transcatheter arterial chemoembolization; OS, overall survival; PFS, progression-free survival; HBV, Hepatitis B; BCLC, Barcelona Clinic Liver Cancer; AFP, alpha-fetoprotein.

\section{Figure 5}

Kaplan-Meier analysis of overall and progression free survival in patient subgroups. (A) OS among patients with BCLC-C; (B) OS among patients with MVI; RT, radiotherapy; PD1, monoclonal antibody 
against programmed cell death; TACE, transcatheter arterial chemoembolization; OS, overall survival; PFS, progression-free survival; BCLC-C, Barcelona Clinic Liver Cancer C stage; MVI, macrovascular invasion; AFP, alpha-fetoprotein. 